\title{
Internação de idosos por causas externas em um hospital público de trauma*
}

Recebido em: 16/02/2011

Aceito em: 16/11/2011

\author{
Silvana Cláudia Bastos de Melo \\ Sandra Maria Cezar Leal ${ }^{2}$ \\ Mara Ambrosina de Oliveira Vargas ${ }^{3}$
}

A Classificação Internacional de Doenças denomina como Causas Externas (CEs) os agravos à saúde resultantes de agressões, acidentes, traumas e lesões. São eventos acidentais ou intencionais. O objetivo deste estudo foi caracterizar as internações de idosos, decorrentes de CEs, em um hospital de pronto socorro de Porto Alegre, no período de julho a dezembro de 2005. Pesquisa retrospectiva. Os sujeitos foram idosos internados. A coleta ocorreu no prontuário dos mesmos. A análise foi realizada com auxílio do software Epi-Info e as variáveis, representadas por tabelas. No período do estudo, 402 idosos foram hospitalizados e as mulheres representaram o percentual de 60,2\%, entretanto, na faixa etária de 60 a 70 anos, os homens foram a maioria. As quedas foram os agravos mais frequentes, a maioria ocorrendo no domicilio. A fratura proximal de fêmur representou $43 \%$, seguida de traumas cranioencefálicos , $14,4 \%$. A maioria dos idosos $(69,2 \%)$ era procedente de Porto Alegre. Considera-se que agravos decorrentes de CEs, principalmente entre os idosos, representam um problema de saúde pública que pode ser evitado. Entende-se que são produzidos socialmente e que ações educativas podem reduzir suas causas e consequências.

Descritores: Idoso, Causas Externas, Hospitalização.

\section{Hospitalization of elderly people due to external causes in a trauma public hospital}

The International Classification of Diseases called external causes the health problems resulting from abuse, accidents, traumas and injuries. Events are accidental or intentional. The aim of this study was to characterize the hospitalization of the elderly, resulting from ECs in a hospital emergency room, Porto Alegre, in the period from July to December 2005. Retrospective survey. The subjects were hospitalized elderly people. Collection occurred in their medical records. The analysis was performed using Epi-Info and the variables represented by tables. During the study period 402 elderly people were hospitalized and the percentage of women represented $60.2 \%$, however, aged 60 to 70 years, men were the majority. Falls were the most frequently injuries occurring at home. The proximal femur fractures accounted for $43 \%$ followed by head trauma $14.4 \%$. Most seniors (69.2\%) came from Porto Alegre. It is considered that damages resulting from ECs especially among the elderly represent a public health problem that can be avoided. It is understood that they are socially produced and that educational activities can reduce the causes and consequences.

Descriptors: Elderly, Injury, Hospitalization.

\section{Hospitalización de los ancianos por causas externas en un hospital público de trauma}

La Clasificación Internacional de Enfermedades llamada causas externas (FB), los problemas de salud resultantes de abusos, accidentes, traumas y lesiones. Los eventos son accidentales o intencionales. El objetivo de este estudio fue caracterizar la hospitalización de los ancianos, como consecuencia de EC en una sala de emergencias de un hospital, Porto Alegre, en el período de Julio a Diciembre de 2005. Estudio retrospectivo. Los sujetos hospitalizados tenían edad avanzada. La colecta se produjo en el mismo gráfico. El análisis se realizó con Epi-Info y las variables representadas por tablas. Durante el período de estudio 402 ancianos fueron hospitalizados y el porcentaje de mujeres representa el 60,2\%, sin embargo, de entre 60 y 70 años, los hombres eran la mayoría. Las caídas fueron las más frecuentes, las lesiones ocurren en casa. Las fracturas de fémur proximal representaron el $43 \%$, seguido por el 14,4\% los traumatismos craneales. La mayoría de las personas mayores (69,2\%) procedían de Porto Alegre. Se considera que los daños resultantes de la EC, especialmente entre los ancianos representan un problema de salud pública que puede ser evitado. Se entiende que se producen socialmente y que las actividades educativas pueden reducir las causas y consecuencias.

Descriptores: Anciano, Lesiones, Hospitalización.

\section{INTRODUÇÃO}

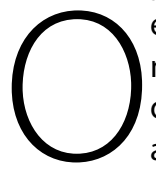
envelhecimento populacional é um fenômeno mundial crescente e ocorre de acordo com o estágio de desenvolvimento econômico dos países. Para a Organização Mundial da Saúde (OMS), idosos são os indivíduos a partir dos 60 anos de idade, para países em desenvolvimento, e de 65 anos de idade para os países desenvolvidos $^{(1)}$.
No Brasil, em 2000, a população maior de 65 anos representava $5 \%$. Atualmente, a projeção estimada é de que em 2050 os idosos representem $18 \%$ da população brasileira ${ }^{(2)}$.

A fase do envelhecimento é influenciada por fatores biológicos, doenças e agravos causados por causas externas (CEs), como quedas, acidentes domésticos, de trânsito, homicídios e suicídios(3).

1 Enfermeira graduada pela UNISINOS.

2 Enfermeira. Mestre e Doutora em Enfermagem EENF/UFRGS. Professora da Graduação em Enfermagem da UNISINOS. Atua no Hospital de Pronto Socorro de Porto Alegre.

3 Enfermeira. Doutora em Enfermagem. Professora Adjunto da UFSC.

*Artigo extraído de monografia de final de Curso de Graduação em Enfermagem da UNISINOS. 
A Classificação Internacional de Doenças da OMS denomina como CEs os agravos à saúde resultantes de agressões, acidentes, traumas e lesões. Podem ser acidentais (quedas, envenenamentos, afogamentos, acidentes de trânsito, de trabalho e outros tipos) e intencionais (agressões, lesões autoprovocadas, homicídios e suicídios). Quanto à natureza da lesão, englobam envenenamentos, ferimentos, fraturas e queimaduras, entre outros ${ }^{(4)}$. Provocam forte impacto na morbimortalidade da população, gerando preocupação para a sociedade, e configuram um problema de Saúde Pública de grande magnitude e transcendência ${ }^{(5)}$.

As CEs representam a sexta causa de morte entre os idosos, perdendo para doenças respiratórias, endócrinas, digestivas, infecciosas e tumores ${ }^{(6)}$. Entre as principais causas de mortes de idoso, decorrentes de CEs, a queda é o mais importante motivo de demanda por serviços de emergência, sendo que a mortalidade aumenta a partir dos 70 anos, atingindo um ápice entre os 80 e 89 anos. Estima-se que após os 85 anos a cada cinco quedas, uma leva a óbito $^{(7)}$. Estas frequentemente ocorrem entre o quarto e o banheiro, no ambiente doméstico; nas ruas e avenidas e dentro do transporte coletivo. Ocorrem, principalmente, devido a enfermidades e fragilidades como a fraqueza muscular, osteoporose, diminuição da visão e da coordenação motora e instabilidade visual e postural, típicas da idade ${ }^{(4)}$. Entretanto, ressalta-se que muitas das situações têm sua intencionalidade invisibilizada pelo argumento de queda acidental.

Os riscos de quedas podem ser minimizados com a adoção de medidas de segurança na área pública e privada ${ }^{(8 ; 3)}$, que visem diminuir as barreiras arquitetônicas.

De outro modo, a promoção da saúde também contribui para a redução desses riscos. Logo, atividades voltadas a grupos sociais e indivíduos, por meio de políticas públicas abrangentes na busca de melhores condições de vida, asseguram aos idosos o prolongamento da existência com manutenção da capacidade funcional, física, mental e da qualidade de vida. Além disso, é preciso garantir atendê-los de maneira integral, com provisão de serviços sociais, condições de moradia, alimentação, transporte e recreação( ${ }^{(9)}$.

Nessa perspectiva, os idosos e seus familiares podem ser considerados como agentes ativos na construção do cuidado à saúde, de forma a atuar na minimização dos efeitos incapacitantes e na diminuição do risco de óbito precoce ${ }^{(10)}$.

Dentre os acidentes de trânsito, o atropelamento é a causa que mais mata idosos no Brasil. Isso se dá pela combinação da dificuldade de movimento do idoso, da falta de respeito impingido por motoristas, e pela negligência do Poder Público ${ }^{(4)}$.

Quanto às causas externas intencionais, destacam--se três questões de relevância relacionadas ao idoso: a negligência social associada à omissão do Estado quanto à necessidade de investir em programas para a proteção do idoso; a violência institucional mais frequente nas instituições de longa permanência; o transporte público e o trânsito, que trata da circulação do idoso pela cidade, e do design dos ônibus(6).

As violências contra os idosos são abrangentes e disseminadas no país, mas a maioria não chega aos serviços de Saúde, ficando naturalizados no cotidiano das relações familiares e nas formas de negligência social e das políticas públicas(6).

Os maus-tratos podem ocorrer por meio de abuso físico, emocional, psicológico, sexual, financeiros e/ou negligência, e sempre acontece numa relação onde existe confiança(4). Prevalecem em mulheres com 75 anos ou mais; viúvas, física ou emocionalmente dependentes, que residem com seus familiares, um dos quais é o seu agressor/abusador ${ }^{(11)}$.

Ressalta-se que a violência doméstica e os maus-tratos a idosos devem ser entendidos no contexto da violência socioestrutural em que o indivíduo está inserido, independente de raça, gênero, classe social, idade ou sexo. São percebidos de diferentes formas entre culturas e sociedades ${ }^{(11)}$. O que não justifica a naturalização do fenômeno.

Negligência é a recusa do responsável pelo idoso em proporcionar os cuidados de que ele necessita. Está presente no ambiente doméstico e institucional. Dela originam-se lesões, traumas físicos, emocionais e sociais. Dentre a especificidade de gênero, dentro de casa, as mulheres são mais maltratadas, e, na rua, os homens são as vítimas. Essa situação causa depressão, desordem pós-traumática e negação das situações de maus-tratos ${ }^{(6)}$

De acordo com os Indicadores e Dados Básicos para a Saúde no Brasil (IDB), em 2008, registraram-se 122.014 internações de idosos por CE SIH/SUS. Dentre elas, $49 \%$ foram decorrentes de quedas e $7 \%$ acidentes de trânsito e transporte, com ênfase em atropelamentos ${ }^{(12)}$. Dentre as principais causas de morte de idosos no Brasil, no ano de 2007, as CEs ocuparam o quinto lugar, representando $4 \%$ do total. Em 2007, morreram 18.946 idosos por CEs no país. No RS, o ano de 2007 ocupou a quarta posição em mortes de idosos por violências e acidentes ${ }^{(13)}$.

Entretanto, os registros relacionados aos óbitos eà morbidade referem-se aos casos de lesões e traumas de indivíduos que são atendidos nos serviços de Saúde ou de Segurança Pública. Vale dizer que muitas situações de óbitos decorrentes de acidentes e violências ficam subnotificados, tendo como causa do óbito uma complicação oriunda do trauma ${ }^{(4)}$.

Tendo em vista o crescimento dos acidentes e violências sofridas pelos idosos, buscou-se, neste estudo, identificar quais são os agravos decorrentes de CEs encontrados nos idosos internados em um hospital público de referência no atendimento ao trauma.

O objetivo geral foi caracterizar as internações de pacientes 
idosos, decorrentes de agravos por CEs, no Hospital de Pronto Socorro de Porto Alegre, no período de $1^{\circ}$ de julho a 31 de dezembro de 2005.

O objetivo específico foi caracterizar os idosos internados em decorrência de agravos por CEs quanto ao sexo, idade, procedência, tipo de agravo e local da ocorrência, no período do estudo.

\section{MÉTODO}

O estudo é do tipo transversal retrospectivo, realizado em um hospital público de referência no atendimento ao trauma. Os sujeitos foram todos os idosos com idade maior ou igual a 60 anos hospitalizados por agravos decorrentes de CEs, no Hospital de Pronto Socorro de Porto Alegre/ RS, no ano de 2005.

A coleta dos dados foi realizada nos prontuários, nos quais os registros eram precários. Para a maioria não foi possível identificar a situação na qual ocorreu o trauma. O Serviço de Documentação e Estatística do Hospital forneceu uma listagem com 456 idosos hospitalizados por causas externas no período do estudo, dos quais 50 participantes foram excluídos, por não haver em seus prontuários registros relacionados ao agravo. O número de participantes totalizou 402 idosos.

Os dados obtidos foram descritos, agrupados e analisados com índices frequenciais absolutos e relativos, com auxílio do software Epi-Info 3.4.2.

Seguiram-se as normas preconizadas pela Resolução no 196/96 (14). Implementou-se a coleta dos dados, após a autorização da Instituição em estudo e aprovação do projeto de pesquisa pelo Comitê de Ética em Pesquisa da Secretaria Municipal de Saúde de Porto Alegre, parecer CEP 116 processo n 001.051333.06.6.

\section{RESULTADOS E DISCUSSÃO}

No período de julho a dezembro de 2005 , foram internados no HPS, 402 idosos por agravos decorrentes de $C E$, representando uma prevalência de 0,38 , considerando-se que o total de usuários hospitalizados, no mesmo período, com idade $\geq$ a 60 anos foi de 1052 idosos.

Os tipos de agravos mais frequentes foram a queda da própria altura com 211 internações (52,50\%), outros tipos de quedas com $82(20,40 \%)$ e acidente trânsito com 58 (14,40\%) idosos hospitalizados. Vale ressaltar que associados, os tipos de quedas atingem o percentual de 72,9\%, totalizando 293 internações de idosos.

As quedas de idosos são decorrentes da perda total do equilíbrio postural, e podem estar relacionadas à insuficiência súbita dos mecanismos neurais e osteoarticulares envolvidos na manutenção da postura. Por se tratar de um evento multifatorial e heterogêneo, pode estar relacionado à síndrome geriátrica $(\mathrm{SG})^{(3)}$. A queda pode afetar a capacidade funcional por estar associada a modificações anatômicas do processo de envelhecimento e a outras patologias. O risco de cair aumenta com o avançar da idade, situando a SG como um problema de Saúde Pública, devido ao aumento do número de idosos na população e à sua maior longevidade ${ }^{(15)}$.

A queda é responsável pela perda da autonomia e da independência do idoso, mudando a dinâmica familiar e trazendo dificuldades de interação com a comunidade onde está inserido(16).

Neste estudo, os acidentes de trânsito representaram 14,4\% (58) de percentual, a maioria relacionada a atropelamentos. Os atropelamentos, os acidentes de trânsito e de transporte são a primeira causa de mortes de idosos, ocorrendo frequentemente devido à inabilidade/descuido do motorista, associado às próprias limitações dos idosos e constituindo, assim, demanda significativa por atendimento de idosos nas emergências hospitalares ${ }^{(6)}$.

$\mathrm{Na}$ análise das internações resultantes de agravos por CE dos idosos deste estudo, identificou-se que as quedas e os acidentes de trânsito foram as causas mais frequentes. Tiveram variações em relação à faixa etária e ao sexo, ocorrendo mais entre os homens com 60 a 70 anos. Entretanto, nos idosos com mais de 70 anos foi mais frequente entre as mulheres, indicando que elas são as principais vítimas a partir dessa idade.

Sugere-se como causa para o maior índice de hospitalizações do sexo feminino a fragilidade das mulheres em relação aos homens, a prevalência de doenças crônicas, a frequência diminuída de atividades externas, a utilização acentuada de drogas, o uso de psicotrópicos, a diminuição da força de preensão, maior exposição a atividades domésticas e a um comportamento de mais atividade assumido pelas mulheres idosas ${ }^{(8)}$. Enfatiza-se também que as mulheres sofrem mais hospitalizações que os homens devido às fraturas por quedas, lembrando que a osteoporose é considerada um dos fatores de risco e tem maior incidência no sexo feminino(17).

Os idosos passam por uma combinação de desvantagens próprias da idade, associadas à falta de respeito e violências impingidas por motoristas e pelo poder público, acarretando as violências no trânsito(4). Considera-se que os acidentes de trânsito são mais frequentes com os homens, por algumas características do sexo masculino, como excesso de autoconfiança e dificuldade para aceitar as limitações com o avançar da idade, entre outras.

Nesta pesquisa, ao relacionar tipo de agravo e local de ocorrência, observou-se que a maior frequência dos agravos ocorre no ambiente doméstico e na via pública, totalizando respectivamente $246(61,2 \%)$ e $83(20,6 \%)$ hospitalizações de idosos. Os dados corroboram as reflexões de pesquisadores ${ }^{(3)}$ sobre resultado de estudos realizado nos EUA, onde $30 \%$ das pessoas com idade superior a 60 anos caem no próprio 
domicílio, sendo acometidos por lesões, traumas ou óbitos, constituindo a etiologia de morte acidental. Incluem-se no risco de quedas do idoso as limitações próprias da idade, o uso de medicamentos, problemas músculoesqueléticos, alterações sensoriais, e ainda problemas ambientais ${ }^{(9)}$.

Muitas vezes, enfermidades como a osteoporose, a instabilidade visual e postural típicas da idade e o processo de envelhecimento do indivíduo, representado pela perda da habilidade de desempenhar tarefas, podem ser indicativos de quedas. Entretanto, a negligência da família em prover adequada proteção ao idoso dentro do domicílio pode ser outro indicativo para os altos índices de quedas domésticas. Assim, a assistência constante dos familiares ou responsáveis é válida para diminuir os agravos ocorridos no domicílio(4).

Acredita-se que um dos fatores relacionados às quedas no domicílio está associado à dificuldade do idoso e da família em perceber como se dá o processo do envelhecimento e o quanto isso limita o idoso (alterações sensoriais como déficit de visão, entre outros).

Na distribuição dos idosos internados no HPS por agravos decorrentes de CEs, quanto ao tipo de agravo e procedência, no período do estudo, identificou-se que a maioria dos idosos hospitalizados é proveniente de Porto Alegre 278 $(69,2 \%)$, cidade na qual localiza-se o Hospital em estudo, seguido da Região Metropolitana $76(18,9 \%)$ e interior do Estado 39 (9,7\%).

A maioria dos agravos ocorre no próprio domicílio, no bairro onde residem, nas localidades próximas onde praticam atividade de lazer ou visitam parente ou amigo ${ }^{(16)}$.

O ambiente físico das vias de acesso das cidades é construído com barreiras arquitetônicas propícias a riscos de quedas eventuais à sociedade, mas principalmente aos idosos ativos, que deslocam seu centro de gravidade no processo de caminhada. $\mathrm{O}$ ambiente apresenta segurança deficiente para a realização das atividades diárias dos idosos, como deambular pelos arredores do domicílio ou ir à casa do vizinho, devido a inclinações que podem levar a uma instabilidade postural, somadas às alterações fisiológicas e patológicas apresentadas pelos idosos, potencializando as quedas ${ }^{(18)}$.

Os acidentes de trânsito atingem os idosos por uma combinação de desvantagens próprias da idade, por estarem mais vulneráveis aos traumas, por terem capacidade reduzida de recuperação, ficando mais tempo hospitalizados com traumatismos graves. A mortalidade é significativamente maior do que aquela dos pacientes mais jovens, devido ao próprio processo de envelhecimento ${ }^{(19)}$.

Quanto ao tipo de lesão dos idosos deste estudo, identificou-se que os tipos mais frequentes são fraturas de colo do fêmur, fraturas trocantéricas e TCE, que representam os percentuais de $28,1 \%$ (113), $14,9 \%$ (60) e $14,4 \%$ (58), respectivamente. Os resultados corroboram os estudos de vários pesquisadores que citam as fraturas como o maior agravo decorrente de quedas, e que se localizam principalmente em membros inferiores, com ênfase para as fraturas proximais de fêmur, seguindo-se os traumatismos intracranianos ${ }^{(4 ; 17)}$.

O tratamento para a fratura de fêmur é cirúrgico, causando medo de cair novamente e restringindo o restabelecimento dos idosos, e muitas vezes dificultando-lhes retomar suas atividades anteriores à queda, como se vestir ou tomar banho sozinho. Essas restrições podem estar associadas também às dores durante a queda e no pós-operatório, acarretando, em muitas situações, restrições definitivas e dependentes ${ }^{(3)}$.

Nesta pesquisa, ao relacionar as lesões e os agravos, constata-se que as fraturas de colo de fêmur e as fraturas trocantéricas são as principais lesões decorrentes de quedas para o idoso, totalizando 158 lesões. OTCE ocupa o segundo principal agravo relacionado às quedas. Tais agravos contribuem para reduzir a mobilidade da pessoa idosa. A maioria das fraturas da extremidade proximal do fêmur é secundária a quedas da própria altura, cuja etiologia é usualmente fatorial, consistindo em uma combinação de comorbidades clínicas, neuropsíquicas, uso de drogas e fatores ambientais. A queda da própria altura pode envolver diversas etiologias relacionadas ao ambiente, tais como piso escorregadio, objetos espalhados no chão; ao condicionamento físico e psíquico; ao uso de medicações e patologias próprias do envelhecimento como a fragilidade óssea causada pela osteoporose. Podem-se, ainda, associar a estas, problemas com o ambiente. Têm como causa mais comum a fratura e, dentre elas, com maior frequência, a fratura do colo do fêmur ${ }^{(3)}$.

\section{CONCLUSÃO}

O estudo quantitativo do tipo transversal limita-se ao recorte de uma determinada situação em um determinado momento. Isso possibilitou caracterizar os idosos internados no HPS, em decorrência de agravos por CEs, e ainda identificar os tipos mais frequentes de hospitalização de idosos neste hospital de trauma.

No período do estudo, foram hospitalizados 402 idosos com agravos decorrentes de causas externas. $\mathrm{Na}$ caracterização relacionada ao sexo, as mulheres representaram o percentual $60,00 \%$. No que se refere à faixa etária, os homens foram mais frequentes entre 60 e 70 anos e as mulheres com idade igual ou maior que 70 anos. Considera-se que mulheres sofrem mais hospitalizações por terem, atualmente, uma longevidade superior aos homens e devido às fraturas por quedas, em geral associadas à osteoporose, fatores de riscos de maior incidência entre elas. As quedas da própria altura constituíram o tipo de agravo 
mais frequente quando associadas a outros tipos de quedas, representando o percentual de 72,9\%. Ainda nesse sentido, ao se relacionar agravos com lesões sofridas pelos idosos, constata-se que a fratura proximal do fêmur, com $28,1 \%$ do percentual, foi o principal tipo de lesão. A maioria ocorreu no próprio domicílio, o que pode indicar que as barreiras arquitetônicas estão espalhadas por todos os lugares, inclusive no ambiente no qual eles vivem, oferecendo riscos à saúde dos idosos e demonstrando que não está havendo proteção contra esse agravo. Ressalta-se que as quedas podem ser acidentais ou intencionais e que profissionais e serviços de saúde devem estar capacitados para identificar situações de maus-tratos, mediante atendimento do idoso em um serviço de saúde com história de queda. Considera-se importante a divulgação de medidas para diminuir os riscos de quedas da própria altura no domicilio, para os idosos, tais como colocar barras no banheiro, pisos antiderrapantes, iluminação adequada, entre outros.
Os acidentes de trânsito com 14,4\% de percentual configuraram o segundo tipo deagravo mais frequente, a maioria relacionada a atropelamentos, causando, principalmente, TCE. Ressalta-se que o processo de envelhecimento e suas consequências, a negligência dos motoristas e o desrespeito às leis de trânsito, entre outros, contribuem para aumentar as estatísticas de atropelamentos. Destaca-se a importância em discutir o problema de trânsito no Brasil, enfatizando maior empenho nas campanhas de educação para o trânsito, a fim de reduzir os riscos de atropelamentos de idosos.

Os resultados encontrados neste estudo contribuem para uma reflexão sobre a fragilidade dos idosos e para a falta de preparo da população quanto ao processo de envelhecimento e suas características. Considera-se que esses agravos são produzidos socialmente e que representam um problema de Saúde Pública que pode ser reduzido com ações solidárias, educativas e de prevenção direcionadas a essa população.

\section{Referências}

1. Pereira RS, Curioni CC, Veras, R. Perfil demográfico da população idosa no Brasil e no Rio de Janeiro em 2002 [Internet]. [citado em 2008 Dez 10]. Disponível: http://www.unati.uerj.br/tse/scielo.php?script=sci_arttext\&pid=S1517. $59282003000100004 \&$ Ing=pt\&nrm=iso

2. Nogueira SL, Geraldo JM, Machado JC, Ribeiro RCL. Distribuição espacial e crescimento da população idosa nas capitais brasileiras de 1980 a 2006: um estudo ecológico [lnternet]. [citado em 2011 Nov 10]. Disponível em: http://www. scielo.br/pdf/rbepop/v25n1/v251a12pdf

3. Fabrício SCC, Rodrigues RAP, Costa Junior ML. Causas e consequências das quedas de idosos atendidos em hospital público. Rev Saúde Pública. 2004;38(1):93-9.

4. Minayo MCS. Violência contra idosos: o avesso do respeito à experiência e à sabedoria. Brasília: Secretaria Especial dos Direitos Humanos; 2004.

5. Ministério da Saúde (BR). Portaria MS/GM no737. Política nacional da redução de morbimortalidade por acidente e violência. Brasília: Ministério da Saúde; 2002. 6. Minayo MCS. Violência contra o idoso: relevância para um velho problema. Cad Saúde Pública. 2003;19(3):783-91.

7. Pereira SRM. Repercussões sócio-sanitárias da epidemia das fraturas de fêmur sobre a sobrevivência e a capacidade funcional do idoso [Tese]. Rio de Janeiro: Escola Nacional de Saúde Pública, Fundação Oswaldo Cruz; 2003.

8. Florença A, Keiko B, Maria C, Correa F, Pettine K. Fratura do colo do fêmur [Internet]. [citado em 2010 Jun 11]. Disponivel em: http://www.wgate.com.br/ fisioweb/traumato.asp

9. Mathias TAF, Jorge MHPM, Andrade OG. Morbimortalidade por causas externas na população idoso residente no município da regiẫo sul do Brasil. Rev Latinoam Enferm. 2006;14(1):17-24.

10. Carboni RM, Reppetto MA. Uma reflexão sobre a assistência à saúde do idoso no Brasil [lnternet]. [citado em 2010 Jan 09]. Disponivel em: http://www.fen.ufg. br/revista/v9/n1/v9n1a20.htm.
11. Pasinato MT, Camarano AA, Machado L. Idosas vítimas de maus-tratos domésticos: estudo exploratório das informações dos serviços de denúncia [Internet]. [citado em 2008 AGO 06]. Disponível em: http://www.abep.nepo. unicamp.br/encontro2006/docspdf/ABEP2006_234.pdf

12. DATASUS. Indicadores de morbidade e fatores de risco. D.14 Proporção de internações hospitalares (SUS) por causas externas 2008 [Internet]. [citado em 2011 Out 10]. Disponível em: http://tabnet.datasus.gov.br/cgi/deftohtm. exe?idb2009/d14.def

13. DATASUS. Indicadores de mortalidade. C4 Mortalidade proporcional por grupo de causas 2009 [Internet]. [citado em 2011 Out 10]. Disponível em: http:// tabnet.datasus.gov.br/cgi/deftohtm.exe?idb2009/c04.def

14. Ministério da Saúde (BR). Conselho Nacional de Saúde. Resolução 196/96 sobre pesquisa envolvendo seres humano. Brasília: Diário Oficial da União, 10 out 1996. p.21082-5.

15. Perracini MR, Ramos LR. Fatores associados a quedas em uma corte de idosos residentes na comunidade. Rev Saúde Pública. 2002;36(6):709-16.

16. Silva TM, Nakatani AYK, Souza ACS, Lima MCS. A vulnerabilidade do idoso para as quedas: análise dos incidentes críticos [Internet]. [citado em 2008 Ago 06]. Disponível em: http://www.fen.ufg.br/revista/v9/n1/v9n1a22.htm

17. Gawryszewski VP, Jorge MHPM, Koizumi MS. Mortes e internações por causas externas entre os idosos no Brasil: o desafio de integrar a saúde coletiva e atenção individual. Rev Assoc Med Bras. 2004;50(1):97-103.

18. Meira EC, Reis LA, Mello IT, Gomes FV, Azoubel R, Reis LA. Risco de quedas no ambiente físico domiciliar de idosos [Internet]. [citado em 2007 Mar 02]. Disponível em: http://www.unati.uerj.br/tse/scielo.php?script=sci arttext\&pid=\$1517-59282005000300006\&lng=pt\&nrm=iso 19. Silveira R, Rodrigues RAP, Costa Júnior ML. Idosos que foram vítimas de acidentes de trânsito no município de Ribeirão Preto-SP, em 1998. Rev Latinoam Enferm. 2002;10(6):765-71. 